\title{
A LOW-NOISE LOW-POWER SECOND-ORDER COMPENSATED CMOS BANDGAP REFERENCE
}

\author{
Sigit Yuwono', Arie van Staveren² \\ ${ }^{1}$ Department of Electrical Engineering, Sekolah Tinggi Teknologi Telkom, Bandung-Indonesia \\ ${ }^{2}$ Electronics Research Laboratory -Delft University of Technology, The Netherlands \\ ${ }^{1}$ yuwono@stttelkom.ac.id, ${ }^{2}$ A.vStaveren@ewi.tudelft.nl
}

\begin{abstract}
The design of a low-noise and low-power second-order bandgap reference voltage source using a linear combination of two base-emitter voltages with only one scaling factor is treated. The design takes into account the temperature dependency of the resistors and the finite current gain of BJT's. The circuit is integrated in a CMOS process. The output voltage is approximately $140 \mathrm{mV}$ with an average temperature dependency of $22.5 \mathrm{ppm} / \mathrm{K}$ in the range of $0^{\circ} \mathrm{C}$ to $120^{\circ} \mathrm{C}$. Its equivalent output noise voltage is $57.6 \mathrm{nV} / \sqrt{\mathrm{Hz}}$. The total current consumption is about $115 \mu \mathrm{A}$ from a $2 \mathrm{~V}$ voltage-supply.
\end{abstract}

Keywords: bandgap reference, negative feedback, systematic design.

\section{Introduction}

A linear combination of two base-emitter voltages of BJT's, whose collector biasing currents exhibit different temperature dependencies, is sufficient to perform temperature dependency compensation. If one of the scaling factors is chosen to be one then the reference voltage at the output of the bandgap reference becomes technologydetermined and has first and second-order frequency temperature.

This paper describes the design of such a bandgap reference circuit with only one scaling factor and optimal noise behaviour. The design is going to be implemented on a CMOS process, therefore the reference BJT's are of the substrate type. The design follows a systematic design approach which is concerned with optimal noise behaviour.

\section{The Basic Theory and the Basic Architecture} of the Bandgap Reference

Figure $1(a)^{[10]}$ is a general topology of a second-order bandgap reference. Because $a_{l}$ is a constant, if $a_{1}$ is shifted after the adder, Figure 1(b), the temperature dependency of the output voltage is unchanged. Thus, $a_{1}$ can be removed by setting it as unity as in Figure 1(c). Figure 1(c) shows that now:

$$
V_{R E F}=V_{B E 1}+a_{2} V_{B E 2}
$$

which is a linear combination of two base-emitter voltage. Writing the base-emitter voltage as a Taylor series around a reference temperature $T_{r}{ }^{[10]}$ results in:

$$
\begin{aligned}
V_{B E m}(T)= & V_{B E m}\left(T_{r}\right)+\left[V_{B E m}\left(T_{r}\right)-V_{G}^{\prime}(0)_{m}\right] \frac{T-T_{r}}{T_{r}}+ \\
& B_{2}\left(\theta_{m}\right)\left(\frac{T-T_{r}}{T_{r}}\right)^{2}+(\text { higher order terms })
\end{aligned}
$$

where:

$$
\begin{aligned}
& B_{2}\left(\theta_{m}\right)=\left[\alpha_{2} T_{r}^{2}-\frac{k T_{r}}{q}\left(\eta-\theta_{m}\right) \frac{1}{2}\right] \\
& V_{G}^{\prime}(0)_{m}=V_{G}\left(T_{r}\right)+\alpha_{1} T_{r}-\left(\eta-\theta_{m}\right) \frac{k T_{r}}{q}
\end{aligned}
$$

in which $\alpha_{1}$ and $\alpha_{2}$ are the first and the second order Taylor coefficient of the bandgap voltage $V_{G}(T)$ at $T_{r}$, respectively, $m$ is the number of BJT generating the base-emitter voltage, $q$ is the electron charge, $k$ is Boltzmann constant, $\theta_{m}$ is the order of the temperature dependency of $\mathrm{BJT}_{\mathrm{m}}$ 's biasing current $\left(\theta_{m}=1\right.$ means a PTAT current and $\theta_{m}=0$ means a constant current), and $\eta$ is the order of the temperature dependency of the saturation current of the BJTs. Substituting $V_{B E m}$ in Equation (1) with of equation (2) results in ${ }^{[10]}$ :

$$
\begin{aligned}
& V_{R E F}=V_{G}^{\prime}(0)_{1}+a_{2} V_{G}^{\prime}(0)_{2} \\
& a_{2}=-\frac{B_{2}\left(\theta_{1}\right)}{B_{2}\left(\theta_{2}\right)}
\end{aligned}
$$

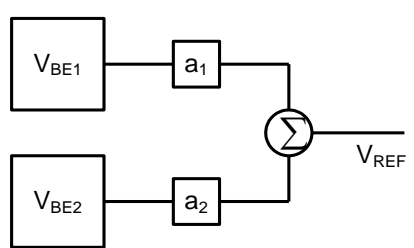

(a)

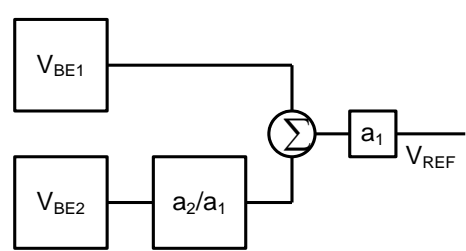

(b)

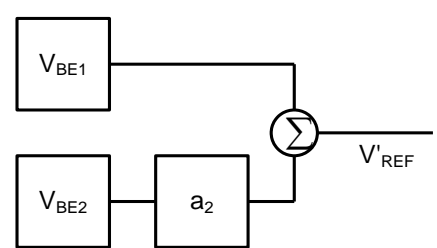

(c)

Figure 1. Second-Order Temperature Compensation:

(a) general architecture, (b) shifting $a_{1}$ through the adder, (c) $a_{l}$ is set to unity. 
Thus $a_{2}$ is determined by the second order temperature dependency compensation. Thus $V_{R E F}$, which is determined by equation (4a) and (4b), is actually determined by the process selected, represented by $\eta$. For AMS CUP (a $0.60 u$ m CMOS technology) $\eta=5.53$. Using Varshini's bandgap energy model ${ }^{[10]}$ and setting $\theta_{1}=1$ and $\theta_{2}=0$, they are obtained: $a_{2}=-0.87$ and $V_{R E F}=146.12 \mathrm{mV}$.

Figure 2 shows the remaining third order temperature dependency of $V_{R E F}$. The mean temperature dependency is approximately 0.6 $\mathrm{ppm} / \mathrm{K}$

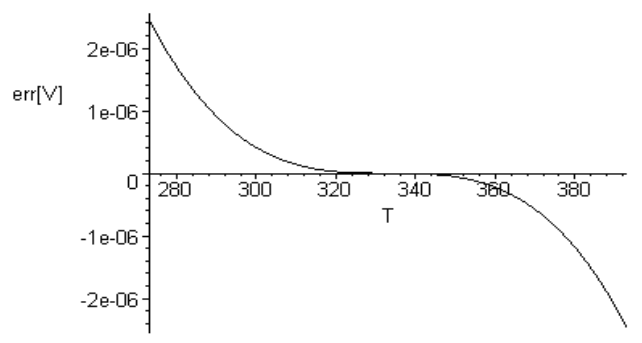

Figure 2. The Remaining $3^{\text {rd }}$-order Temperature Dependency of $V_{R E F}$.

\section{Technology Consideration}

There are two process-dependent factors which also influences the temperature dependency of the bandgap reference circuit.

In $A M S C U P$ process the available BJT is of PNP type so the biasing current will be more convenient expressed in the emitter current rather than in the collector current. Because the current gain, $\beta$, of the BJT available is finite, the emitter current also supplies the base current. This base current gives additional temperature dependency to the base-emitter voltage. The error due to the basecurrent is expressed as ${ }^{[9]}$ :

$$
\Delta V_{B E}(T)=-V_{T} \ln \left[1+\frac{1}{\beta\left(T_{r}\right)} \cdot\left(\frac{T}{T_{r}}\right)^{-Х T B}\right]
$$

where $X T B$ is the order of the temperature dependency of base-current.

Another factor is the resistor used for driving the biasing current experiences also a temperature dependency. This dependency contributes additional temperature dependency to the baseemitter voltage. AMS CUP process only supplies the first order temperature dependency coefficient for its resistor, the error it contributes is ${ }^{[1]}$ :

$$
\Delta V_{B E}\left(T, c_{1}\right)=-V_{T} \cdot \ln \left[1+c_{1}\left(T-T_{r}\right)\right]
$$

where $V_{T}=\frac{k T}{q}$.

By expanding Equation (5) and (6) into its Taylor series, the additional first and second order temperature dependency can be found. Adding these additional temperature dependencies into equation (3) and (4a) appropriately will result in a shift in value of $a_{2}$, which in return also shifts the value of
$V_{\text {REF }}$. The new values after taking these errors into account are: $a_{2}=-0.88$ and $V_{R E F}=134.67 \mathrm{mV}$.

\section{Implementation of the Circuit}

Figure 1(c) is the basic block of the bandgap reference which is going to be implemented. Its output voltage is expressed in equation (1).

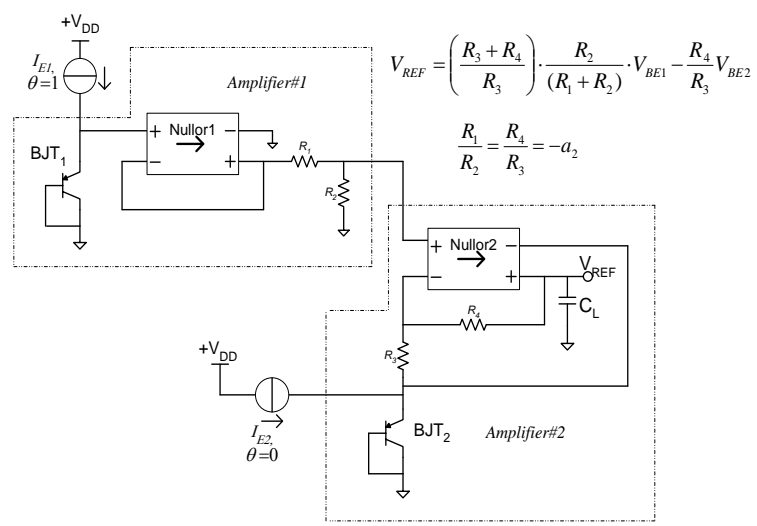

Figure 3. The Bandgap Reference at Nullor Level

\subsection{The Basic Circuit Diagram}

Figure 3 is the bandgap reference at nullor level which implements Figure 1(c). The output of Figure 3 is expressed as:

$$
V_{R E F}=\left(\frac{R_{3}+R_{4}}{R_{3}}\right) \cdot \frac{R_{2}}{\left(R_{1}+R_{2}\right)} \cdot V_{B E 1}-\frac{R_{4}}{R_{3}} V_{B E 2}
$$

To suit Equation (1) these conditions are required:

$$
\frac{R_{4}}{R_{3}}=-a_{2}
$$

and

$$
\frac{R_{1}}{R_{2}}=-a_{2}
$$

\subsection{BJT Biasing}

In Figure 3, $\mathrm{BJT}_{1}\left(\mathrm{BJT}_{2}\right)$ is biased at a PTAT (constant) current level.

The minimum noise voltage at the output of the bandgap reference only due to the noise of the reference transistors, equals ${ }^{[10]}$ :

$$
\bar{v}_{n, e q}=10^{-10} V_{R E F} \sqrt{\frac{B}{I_{M A X}}}
$$

where $I_{M A X}=I_{C 1}+I_{C 2}$, the sum of the collector currents of transistor $B J T_{1}$ and $B J T_{2}$ and $B$ is the bandwidth, chosen $1 \mathrm{kHz}$. Equation (10) then gives approximately $I_{M A X}=5 n$ A.

Because $a_{l}=1$ in this design, for optimal noise behavior following condition should be fulfilled ${ }^{[10]}$ :

$$
\frac{1}{a_{2}}=-\frac{I_{C 1}}{I_{C 2}}
$$


The saturation current of $\mathrm{BJT}_{1} I_{S 1}$ is set equal to of the minimum sized BJT in AMS $0.6 \mu \mathrm{m}$ CUP process while the saturation current of $\mathrm{BJT}_{2} I_{S 2}$ is set by using $N$ times of the minimum sized BJT, in other words, $I_{S 2}=N^{*} I_{S 1}$. The relationship of all these parameters and requirements is given as $^{[3]}$ :

$$
I_{C 2}=I_{S 1} \exp \left\{\frac{1}{\left(1+a_{2}\right)}\left[\frac{q V_{R E F}}{k T_{r}}+\ln \left(-a_{2}\right)+a_{2} \ln (N)\right]\right\}
$$

By plotting $I_{C 2}$ as a function of $N$, a convenient $I_{C 2}$ is found when $N=12$, which gives these values:

$$
I_{C 1}=6.35 \mu \mathrm{A} \text { and } I_{C 2}=5.59 \mu \mathrm{A} \text {. }
$$

Consequently $I_{M A X}=11.84 \mu \mathrm{A}$, much higher than the minimum current found with Equation (10). Putting $I_{C 1}$ and $I_{C 2}$ into following equation:

$$
I_{C}(T)=I_{S}(T)\left[\exp \left(\frac{q V_{B E}}{k T}\right)-1\right]
$$

gives: $V_{B E 1}=572.35 \mathrm{mV}$ and $V_{B E 2}=497.37 \mathrm{mV}$.

Because in CUP process the available BJT is vertical PNP BJT, biasing current is more convenient to be expressed in its emitter current. For CUP process $\beta\left(T_{r}\right)=17.29$, thus:

$$
I_{E 1}=6.71 \mu \mathrm{A} \text { and } I_{E 2}=5.91 \mu \mathrm{A} \text {. }
$$

\subsection{Developing the Bandgap Reference at Circuit Level}

To develop the bandgap reference more systematically the basic circuit in Figure 3 is divided into two amplifiers, i.e.: Amplifier\#1, includes $\mathrm{BJT}_{1}$, Nullor $1, R_{1}$ and $R_{2}$, and Amplifier\#2, includes $\mathrm{BJT}_{2}$, Nullor $1, R_{3}$ and $R_{4}$.

The nullors consist of two stages of amplifier in order to achieve relatively high open loop gain. The input stage is a PMOS differential amplifier and the second stage is an NMOS common source amplifier in order to make the circuit operate under a single polarity voltage supply. The restriction in designing the amplifiers is that the current through the resistors must not be higher than its BJT's emitter current.

\subsubsection{Amplifier\#1}

Following the restriction of maximal current through the resistors $R_{1}$ and $R_{2}$ and fulfilling Equation (9) results in:

$$
R_{l}=39.9 \mathrm{k} \Omega \text { and } R_{2}=45.34 \mathrm{k} \Omega \text {. }
$$

A convenient level of drain current and the dimension of PMOS for the input stage of the nullor are found by setting such a way that its noise level is a half of it produced by resistors $R_{1}$ and $R_{2}$. The high-frequency stability of Amplifier\#1 is achieved by adding a $2 p \mathrm{~F}$ capacitor parallel to $R_{2}$ to eliminate the circuit instability at about $9 \mathrm{MHz}$. Another $2 p \mathrm{~F}$ connects the inputs of Nullor1 to keep the voltage at the terminals at the same phase.

\subsubsection{Amplifier\#2}

Doing a similar step as in Section 4.3.1 gives: $R_{3}=32.652 \mathrm{k} \Omega$ and $R_{4}=28.733 \mathrm{k} \Omega$.

Because resistors in Amplifier\#2 are less than in Amplifier\#1, a convenient level of drain current and the dimension of PMOS for the input stage of the nullor are found after following such a way that its noise level equals of it produced by resistors $R_{3}$ and $R_{4}$. The high-frequency instability is found at $1.8 \mathrm{MHz}$ and eliminated by adding a $5 \mathrm{pF}$ capacitor in series with a $40 \mathrm{k} \Omega$ resistor from the drain of NMOS, the second stage amplifier, to the ground.

\subsection{The constant and PTAT current sources}

The constant current is required to biasing $\mathrm{BJT}_{2}$ in Figure 3. In order to make the current generated by the source relatively immune to the disturbance of power source, the voltage to generate the current is fed from the bandgap reference output voltage. Figure 4 shows the nullor-level block diagram of the constant current source which is a transconductance amplifier.

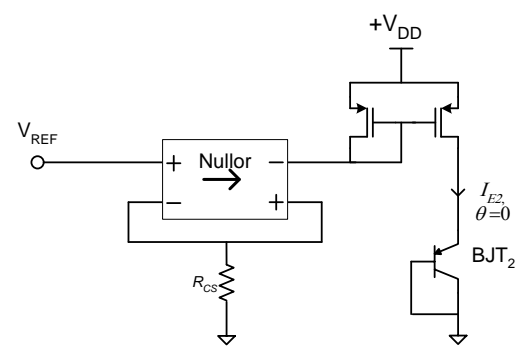

Figure 4. The Constant Current Source and Its Load, $\mathrm{BJT}_{2}$

For this amplifier the high-frequency instability is experienced at $2 \mathrm{MHz}$ and eliminated by adding a $4 \mathrm{pF}$ capacitor in series with a $20 \mathrm{k} \Omega$ resistor from the drain of NMOS, the second stage amplifier, to the ground.

A PTAT current is actually generated from a PTAT voltage source. Generating a PTAT voltage source uses the same equation as generating the bandgap reference, Equation (2). From Equation (2), the constant term is:

$$
V_{P T A T}\left(T_{r}\right)=a_{1} V_{B E 1}\left(T_{r}\right)+a_{2} V_{B E 2}\left(T_{r}\right)
$$

the first-order temperature dependency term is:

$$
V_{1 S T}(T)=\sum_{m=1}^{2} a_{m}\left[V_{B E m}\left(T_{r}\right)-V_{G 1}(0)-\left(\eta-\theta_{m}\right) \frac{k T_{r}}{q}\right] \frac{T-T_{r}}{T_{r}}
$$

and the second and higher-order temperature dependency terms is:

$$
V_{\text {REST }}(T)=\sum_{m=1}^{2} a_{m} \sum_{n=2}^{\infty}\left[\alpha_{n} T_{r}^{n}-\frac{k T_{r}}{q}\left(\eta-\theta_{m}\right) \frac{(-1)^{2}}{2(2-1)}\right] *\left(\frac{T-T_{r}}{T_{r}}\right)^{2}
$$


If the current biasing both BJTs has same temperature dependency, then in order to eliminate the second and higher-order temperature dependency terms:

$$
a_{1}=-a_{2}
$$

This condition changes Equation (15):

$$
V_{1 S T}(T)=V_{P T A T}\left(T_{r}\right) \frac{T-T_{r}}{T_{r}}
$$

which is a PTAT voltage. Exploring Equation (14):

$$
\begin{aligned}
V_{P T A T}\left(T_{r}\right) & =\frac{k T_{r}}{q}\left[a_{1} \ln \frac{I_{C 1}\left(T_{r}\right)}{I_{S 1}\left(T_{r}\right)}+a_{2} \ln \frac{I_{C 2}\left(T_{r}\right)}{I_{S 2}\left(T_{r}\right)}\right] \\
& =\frac{a k T_{r}}{q} \ln \left[\frac{I_{C 1}\left(T_{r}\right)}{I_{S 1}\left(T_{r}\right)} \frac{I_{S 2}\left(T_{r}\right)}{I_{C 2}\left(T_{r}\right)}\right]
\end{aligned}
$$

where $a=a_{1}=-a_{2}$.

If $\mathrm{BJT}_{1}$ is biased with a current which is $N$ times the current biasing $\mathrm{BJT}_{2}$

$$
I_{C 1}\left(T_{r}\right)=N \cdot I_{C 2}\left(T_{r}\right)
$$

and the area of $\mathrm{BJT}_{2}$ is $M$ times the area of $\mathrm{BJT}_{1}$

$$
I_{S 2}\left(T_{r}\right)=M \cdot I_{S 1}\left(T_{r}\right)
$$

then equation (19) becomes:

$$
V_{P T A T}\left(T_{r}\right)=\frac{a k T_{r}}{q} \ln (N \cdot M)
$$

This shows that now the constant ' $a$ ' is not necessary to be set to a certain value. It shows also that the product of $N$ and $M$ have to be larger than one. If the PTAT voltage in equation (19) is across a resistor then the current flowing through the resistor is a PTAT current.

Figure 5 shows a common circuit topology to generate a PTAT current with $a=1$. The voltage across $R_{P T A T}$ is a PTAT voltage. Therefore the current through it is a PTAT current, which biases $Q_{Z}$. This current is mirrored by PMOS pair $M_{Y}$ and $M_{Z}$ to bias $Q_{Y}$. Therefore the biasing current of $Q_{Z}$ and $Q_{Y}$ is of the same temperature behavior. BJT $Q_{Y}$ in Figure 5 also functions as the reference $\mathrm{BJT}_{1}$, as in Figure 3.

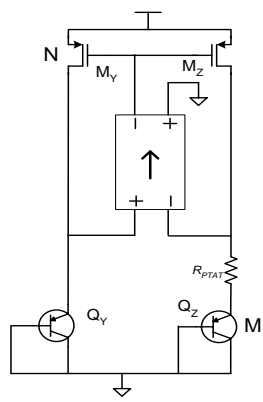

Figure 5. PTAT Current Source

For the frequency behavior analysis, it shows an instability at about $1.7 \mathrm{MHz}$ which is then eliminated by putting a $4 p \mathrm{~F}$ capacitor in series with a $15 \mathrm{k} \Omega$ resistor between the gate of $\mathrm{M}_{\mathrm{Y}}$ and $\mathrm{M}_{\mathrm{Z}}$ and the ground.

\section{Simulation Results}

In this paper three simulation results are represented. They are the output of the PTAT current source circuit (Figure 6), the designed reference voltage (Figure 7) and the effect of the voltage source to the reference voltage (Figure 8).

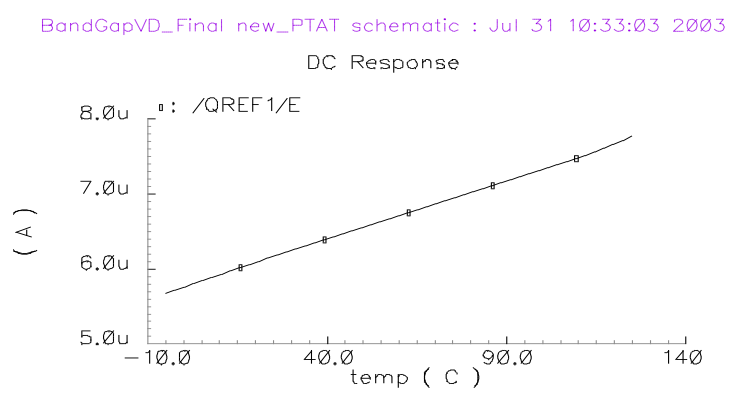

Figure 6. The Generated PTAT Current

The PTAT current source swings linearly from $5.76 \mu \mathrm{A}$ at $0^{\circ} \mathrm{C}$ to $7.66 \mu \mathrm{A}$ at $120^{\circ} \mathrm{C}$ or $15.83 n \mathrm{~A} / \mathrm{K}$. Its output noise level is $0.58 \mu \mathrm{V}$ at $1 \mathrm{kHz}$ and $60^{\circ} \mathrm{C}$.

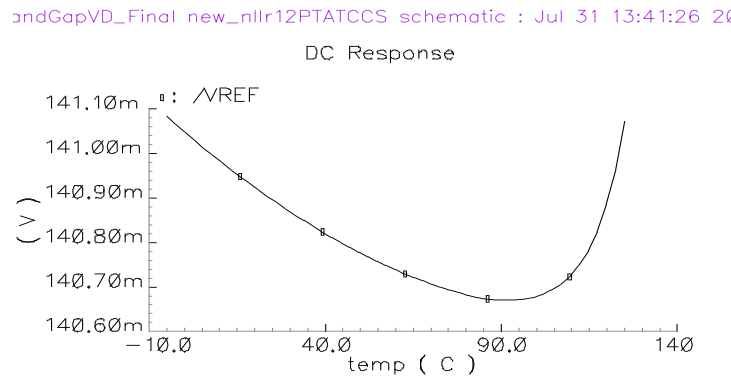

Figure 7. The Output of The Simulated Bandgap Reference.

The mean temperature dependency of the output voltage is about $22.5 \mathrm{ppm} / \mathrm{K}$, in this condition the scaling factors $a_{1}=0.986$ and $a_{2}=0.852$. Its noise level is $1.823 \mu \mathrm{V}$ at $60^{\circ} \mathrm{C}$ and $1 \mathrm{kHz}$.

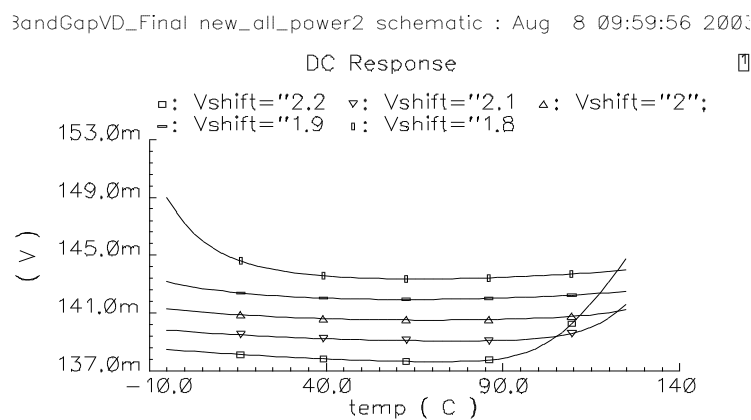

Figure 8. The Bandgap Output Voltage as The Function of The Temperature for Several Level of DC Voltage Supply

From Figure 9 it is shown that as the voltage supply is shifted further from the designed voltage level, the temperature dependency becomes higher. 


\section{Conclusion}

In this research the design of the bandgap reference as well as a PTAT current source have been done and simulated. Due to the use of the substrate BJT, the scaling factor $a_{l}=1$ can be achieved only by a scaling circuit. Therefore, the designed bandgap reference actually has still two scaling factors.

The designed bandgap reference circuit has an output voltage of $140 \mathrm{mV}$ and a temperature dependency of $22.5 \mathrm{ppm} / \mathrm{K}$. Its noise level about $57.6 n \mathrm{~V} / \sqrt{\mathrm{Hz}}$. Its total current consumption is about $115 \mu \mathrm{A}$ from a $2 \mathrm{~V}$ voltage-supply.

\section{References}

[1] Austria Micro Systems, October 1988, 0.6um CMOS CUP Process Parameters, Document\#: 9933011, Revision\#: B.

[2] Austria Micro Systems, October 1988, 0.6um CMOS Design Rules, 7 Digit Document\#: 9931025, Revision\#: 2.0.

[3] Azarkan, van Staveren and Fruett, 2002, A Low-noise Bandgap Reference Voltage Source with Curvature Correction, IEEE International Symposium on Circuit and Systems, 26-29 May 2002. Pages: III-205 - III-208 vol.3. ISBN: 07803-7448-7/2. IEEE.

[4] Gilbert, B. Editors: J. H. Huijsing, R. J. van de Plassche and W. M. C. Sansen,1996, Monolithic Voltage and Current References: Theme and Variations, Analog Circuit Design, Page: $243-$ 352. Dordrecht, The Netherlands, Kluwer.
[5] Howe, Roger T., and Sodini, Charles G., 2003, Microelectronics An Integrated Approach, Lecture $16 \mathrm{~T}-\mathrm{Th}$.

http://www.prenhall.com/howe3/microelectroni $\mathrm{cs} / \mathrm{html} /$ lectures_folder/lectures-tth.html.

[6] Leach, W. Marshall Jr., 1999-2002, EE6416 Low-Noise Electronic Design, Chapter 7 Noise in Diode and BJT's.

http://users.ece.gatech.edu/ mleach/ece6416/.

[7] Leung, Ka Nang, and Mok, Philip K.T., April 2002, A Sub-1-V 15-ppm/ C CMOS Bandgap Voltage Reference Without Requiring Low Threshold Voltage Device. IEEE Journal of Solid-State Circuits. Vol. 37. No. 4.

[8] Van Staveren, Arie, Editors: W.A. Serdijn, C.J.M Verhoeven and A.H.M., 1995, Analog IC Techniques for Low-Voltage Low-Power Electronics, Chapter 5 Integrable DC Sources and References, Delft, Delft University Press.

[9] Van Staveren, Arie, 1999, Structured Electronic Design of Bandgap References and PTAT sources in CMOS, The Netherlands, TUDelft

[10] Van Staveren, Arie, 2000, Structured Electronic Design: High Performance Harmonic Oscillator and Bandgap, Chapter 6 Bandgap References. ISBN 0-7923-7283-2, Dordrecht, Kluwer.

[11] Yuhua Cheng et al., 1996, BSIM3v3 Manual, Barkeley, Department of Electrical Engineering and Computer Science, University of California.

\section{Attachment}

The figure attached is the whole designed circuit. The supplying current sources are not shown.

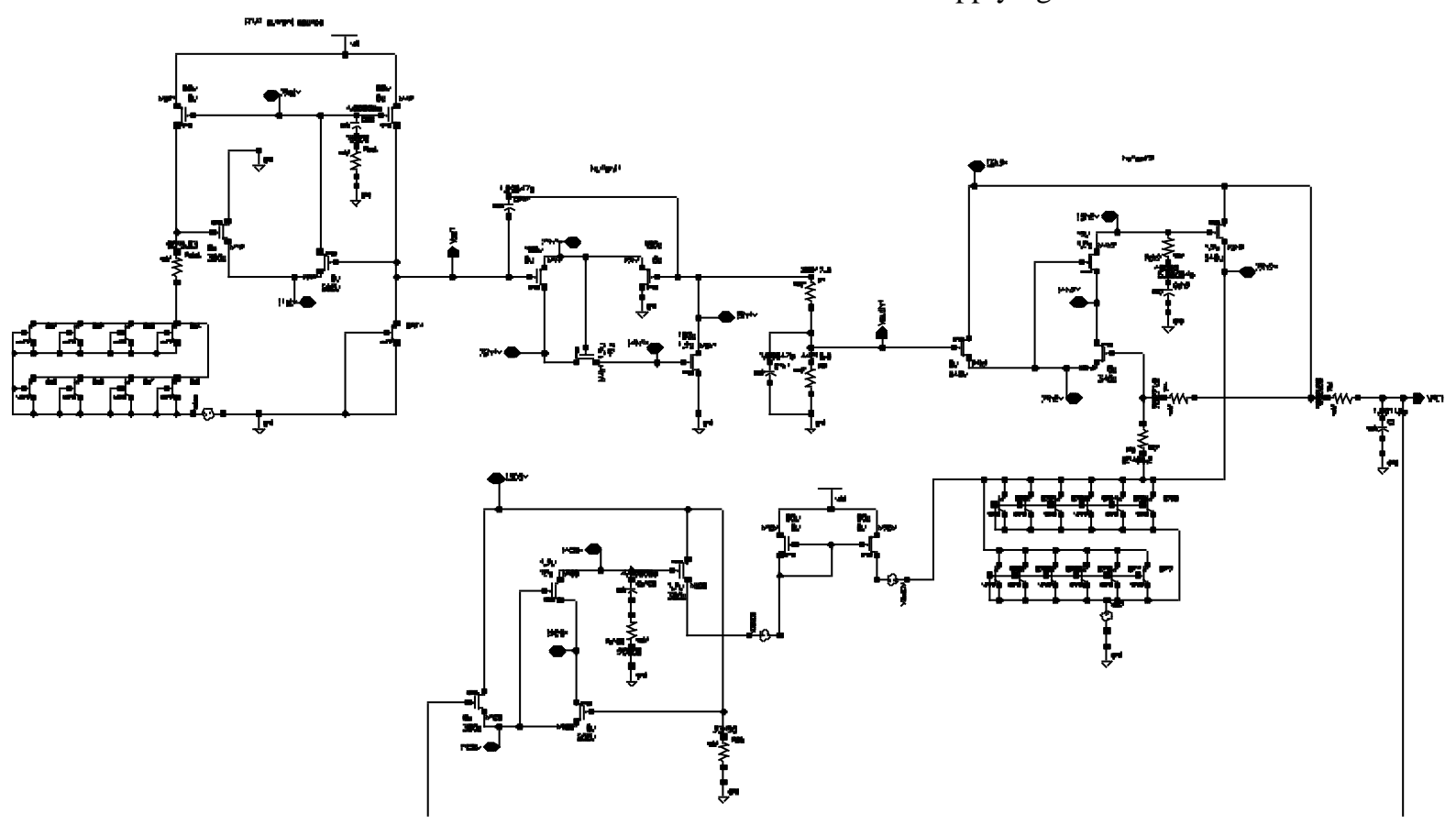

The Schematic of The Designed Bandgap Reference Circuit 